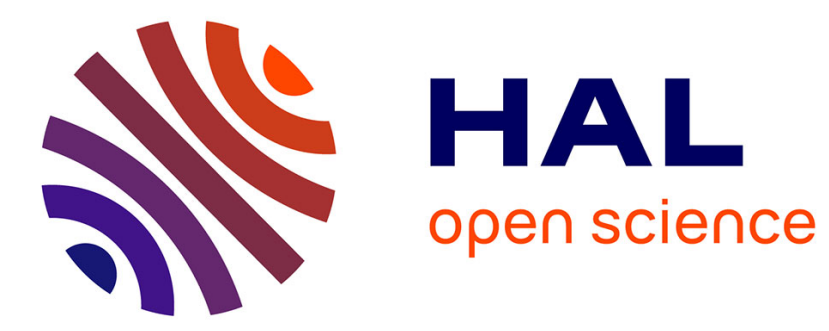

\title{
Energy-Aware Object Tracking Algorithm using Heterogeneous Wireless Sensor Networks
}

Ibtissem Boulanouar, Abderrezak Rachedi, Stéphane Lohier, Gilles Roussel

\section{To cite this version:}

Ibtissem Boulanouar, Abderrezak Rachedi, Stéphane Lohier, Gilles Roussel. Energy-Aware Object Tracking Algorithm using Heterogeneous Wireless Sensor Networks. IFIP Wireless Days Conference, Oct 2011, Niagara Falls, Canada. pp.6. hal-00633034

\section{HAL Id: hal-00633034 https://hal.science/hal-00633034}

Submitted on 28 Mar 2013

HAL is a multi-disciplinary open access archive for the deposit and dissemination of scientific research documents, whether they are published or not. The documents may come from teaching and research institutions in France or abroad, or from public or private research centers.
L'archive ouverte pluridisciplinaire HAL, est destinée au dépôt et à la diffusion de documents scientifiques de niveau recherche, publiés ou non, émanant des établissements d'enseignement et de recherche français ou étrangers, des laboratoires publics ou privés. 


\title{
Energy-Aware Object Tracking Algorithm using Heterogeneous Wireless Sensor Networks
}

\author{
Ibtissem Boulanouar, Abderrezak Rachedi, Stephane Lohier, Gilles Roussel \\ Computer Science Laboratory (LIGM) \\ University of Paris-Est Marne-la-Vallée (UPEMLV) \\ Champs sur Marne, France \\ \{FirstName. LastName\}@univ-mlv.fr
}

\begin{abstract}
Mobile target tracking consists in detecting and locating targets in a surveillance region. Wireless Multimedia Sensor Networks (WMSN) can provide more information about the moving target than classical Wireless Sensor Networks (WSN), but their process and the transmission of multimedia data are costly which reduces the network's lifetime. In this paper, we propose a low-cost new solution for tracking a mobile target called EAOT (Energy Aware Object Tracking). It consists of a distributed cooperative algorithm that runs in heterogeneous Wireless Sensor Networks composed of both scalar and multimedia sensors. The scalar sensors (MS) are equipped with a motion detector; their role is to detect the target and then activate the camera sensors (CS) through message exchanges. We conducted simulations to evaluate the performance of the proposed mechanism in terms of tracking accuracy, number of exchanged messages, energy consumption, and spatial average deviation. The results are compared to two existing solutions: one based on simple camera sensors which always run in active mode and the other one based on the optimal camera node selection (OCNS) to track the target. The obtained results show that for the same number of nodes, EAOT consumes between $2.6 \%$ and $44 \%$ less energy than OCNS depending on the distribution of MS/CS.
\end{abstract}

Keywords-Wireless Sensor Networks, Wireless Multimedia Sensor Network, tracking, mobile target.

\section{INTRODUCTION}

Wireless Sensor Networks (WSN) are small devices networks with resource constraints which can communicate with each other on short distances to harvest and process data.

To make them multifunctional, these sensors have been equipped with small cameras and microphones in order to capture and retrieve multimedia contents. These Wireless Multimedia Sensor Networks (WMSN) allow a wide range of applications such as: intrusion detection, target tracking, habitat and health care monitoring. In addition to the common characteristics shared with WSN, the WMSN have special features: high bandwidth demand, specific QoS requirements, sector sensing range, etc. Moreover, wireless Camera Sensors (CS) unlike classical video systems can be used in both indoor and outdoor environments, where energy and network infrastructure are not available and where no human intervention is possible. They offer a wider panel of applications whether for environmental, industrial or military monitoring [1]. Most recent studies in WMSN focus on increasing the network lifetime by using an energy aware routing protocol, providing Quality of Service (QoS) and security in communications and by placing nodes in order to increase the coverage area.

In this paper, we focus on object tracking in WMSN and more particularly on non-communicating object tracking like human beings, animals, etc. Mobile target tracking consists in retrieving the target's successive coordinates during its moving through the monitoring area. This topic has been well studied in classical WSN, particularly in the case of communicating object tracking. With the emergence of small CMOS cameras, tracking mobile object using video sensor wireless networks has received more attention. The main difficulty is to handle the tradeoff between ensuring the tracking accuracy and maximizing the network lifetime. Indeed, the solution for the best tracking accuracy is to keep all camera sensors active (naive technique), but this is impossible because of energy consumption due to processing and transmission of the multimedia data retrieved. The best solution is to only activate the camera sensors able to localize the target.

We distinguish three main classes of tracking techniques [2]: naive [3], predictive based [4] [5] and dynamic clustering [6]. The naive technique means that all nodes are always in active mode, what maximizes the energy consumption. The second one consists in using a predictive model to anticipate the future position of the target. This technique is not appropriate for some applications like intrusion detection. Indeed, the predictive model used cannot ensure $100 \%$ accuracy of the calculated trajectory. Finally, in the dynamic clustering, a cluster of nodes is selected at each step of the target progression in the area of interest. While the selected cluster of nodes performs the tracking, the other nodes remain in sleeping mode. So this technique saves the network's energy. In order to find a better tradeoff between accuracy and energy saving, we introduce a Heterogeneous Wireless Sensor Network (HWSN) composed of both scalar and camera sensors. The scalar sensor consumes less energy than the multimedia sensor which allows us to keep them always in active mode. We propose an Energy Aware Object Tracking (EAOT) protocol based on message exchange, where the scalar sensors handle the object detection and only activate the camera sensors that can localize the target object. The main characteristics of the proposed EAOT are: 1) immediate 
detection of the target when it enters the monitoring area 2) performing the tracking in a low-cost manner 3) object localization based on three possibilities: the real coordinates, a visual observation of the target and as worst case approximate coordinates.

The rest of this paper is organized as follows: in section II, we review the related work in WMSN research topics. Section III describes the proposed protocol called EAOT. We present and discuss the simulation results obtained from our solution EAOT and other existing solutions in section IV. Finally, section $\mathrm{V}$ concludes the paper and presents our future works.

\section{RELATED WORK}

The mobile object tracking is one of the Wireless Multimedia Sensor Networks (WMSNs) applications. This application consists in locating the mobile target at every step of its progression in the surveillance area. The tracking solutions can be classified in three main categories [2]: naive, predictive based and dynamic clustering. Because of the high cost of the naive technique, it is unreasonable to use such a method in WMSNs where energy is a precious resource.

In the predictive based technique, a predictive model is used to predict the future position of the mobile target. An adapted Kalman Filter is used in [4] to calculate the future sensor utility depending on past data collected. In [5] the authors retrieve the mobility parameters of the target and use an autoregressive model to integrate them and predict the future trajectory.

Dynamic clustering [6] is the most used technique in literature. A cluster of nodes is selected at every step of the evolution of the mobile target in the surveillance region. A cluster head is selected depending on predefined criteria. The advantage of dynamic clustering is that the sensors which are not selected to be cluster members remain in sleeping mode. Thus, this technique allows saving energy.

Papers described here correspond to the third technique .The authors in [7] propose a distributed solution based on node collaboration to select the optimal subset of camera sensors that participate in the target location process. This solution consists of a two phase algorithm that maintains the adequate number of nodes in active mode. When the target is detected by a camera sensor, it broadcasts its location and orientation to the neighbor. Each of them calculates the probability of detecting the target. If the detecting probability reaches a predefined threshold, the concerned node activates its camera to take part in the locating process. This collaborative algorithm is also merging with a wake up algorithm that maintains the adequate number of nodes in active mode. This solution presents a good tradeoff between energy consumption and tracking accuracy but the target can enter the surveillance region without being immediately detected because of the wake up algorithm. SensEye [8] is the first solution that introduces the concept of heterogeneous network. The authors propose three-tier camera sensors; every tier supports a specific task. The first tier assumes target detection and localization while the second one performs target recognition and the last tier assumes target tracking.
SensEye can only be used in indoor environments where energy is available which considerably limits the types of applications where we can use it.

The authors of [9] also used the concept of heterogeneous networks but for different objectives. Indeed, the activation goal is the event coverage, they used the scalar sensors to determine the event boundary and actuate the necessary camera sensors. The main objective is the elimination of data redundancy.

\section{ENERGY AWARE OBJECT TRACKING}

In this section, we present the proposed protocol called Energy Aware Object Tracking (EAOT). Its main objectives are as follows: 1) having a visual observation of the target, which permits its identification 2) having target coordinates to use them for specific applications like intrusion detection 3) saving energy to increase the network's lifetime.

\section{A. Preliminaries}

In this subsection we give some definitions and assumptions. The heterogeneous concept of sensor nodes is described with the characteristics and role of each type of node.

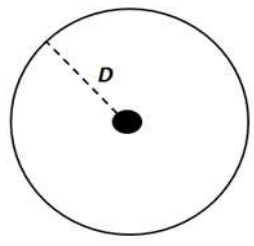

A

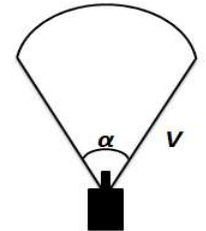

B
Figure 1. FoD and FoV

1) Definition 1: The Motion Sensor (MS) is a wireless scalar sensor equipped with motion detector. Each object located in the Field of Detection (FoD) of MS can be detected. FoD is represented by a circle with radius D (as illustrated in figure 1.A).

2) Definition 2: The Camera Sensor (CS) is a wireless multimedia sensor equipped with both motion detector and video camera. Each object located in CS's Field of View (FoV) can be visually detected. FoV is a CS's directional of view and it is assumed to be a cone with angle $\alpha$ and radius $\mathrm{V}$ (as illustrated in figure 1.B).

The main difference between MS and CS is not only limited to the services but also to the energy consumption. CS requires more energy to run its cameras compared to MS.

3) Definition 3: We introduce the concept of approximate coordinates (x,y). Equations 1 and 2 show how these coordinates can be calculated. 


$$
\begin{gathered}
\boldsymbol{x}=\mathbf{P}_{\mathbf{M S}}\left(\frac{\sum_{i=1}^{N} \mathrm{X}_{i}}{\mathrm{~N}}\right) \\
\boldsymbol{y}=\mathbf{P}_{\mathbf{M S}}\left(\frac{\sum_{i=1}^{N} \mathrm{Y}_{i}}{\mathrm{~N}}\right)
\end{gathered}
$$

Where PMS represents the probability that the target is detected by MS and $\mathrm{N}$ is the total number of MSs that detect the target in the given area at the same time.

The target is detected by MS when it is in its FoD. In other words: the probability PMS that a target at distance RMS is detected is the probability that RMS $\leq \mathrm{D}$, where $\mathrm{D}$ is the circle radius of FoD.

$$
\mathbf{P}_{M S}=P\left(\boldsymbol{R}_{M S} \leq \boldsymbol{D}\right)
$$

In [10], the authors provide a probabilistic analysis of a simple detection system. Using their concept, we can calculate PMS as follows:

$$
\mathbf{P}_{M S}=P\left(\frac{\mathbf{R}_{M S}-\boldsymbol{\mu}}{\sigma} \leq \frac{D-\mu}{\sigma}\right)=\phi\left(\frac{D-\mu}{\sigma}\right)
$$

Where $\mu$ and $\sigma$ are respectively the mean and the standard deviation. $\phi$ is the distribution function of standard normal random variable. PMS decreases while the RMS increases.

We assume the random deployment of scalar and multimedia sensors in the monitoring region. All deployed motion sensors (MS) have the same field of detection radius (FoD). The same assumption is valid for all deployed camera sensors (CS) which have the same field of view (FoV) parameters. Moreover, we assume that the nodes are static, each MS knows its location and each CS knows its location and orientation using one of the existing methods described in literature [11]. A single mobile target at a time is expected to cross the monitored region by taking a random path. Every sensor can communicate with another one as long as it is in its transmission range, independently of the type of sensor. Initially we supposed that all cameras are inactive and the motion detectors are always active.

\section{B. Solution overview}

The proposed solution EAOT is a cooperative protocol based on two types of packets called: DETECTION and LOCALIZATION. The DETECTION packet is sent only by the MS node to inform the neighboring nodes, particularly CS nodes, about the presence of any object in its FoD area. The LOCALIZATION packet is sent only by the CS nodes to the first MS that informs it once they receive a certain threshold $\boldsymbol{M}$ of DETECTION packets from the MS nodes. Both packet types contain the coordinates of sender nodes. In addition, the packet size of DETECTION and LOCALIZATION is similar and equal to $64 \mathrm{~Kb}$.

Figure 2 illustrates an example of object tracking with the proposed solution EAOT. MS1 is the first node that detects the target (the target trail is illustrated by the dashed line). MS1 broadcasts a DETECTION message containing its own coordinates to the nodes which are in its transmission range.
The life span of DETECTION message $\mathrm{T}$ is fixed to 20 seconds, chosen according to the target speed. Once MS2 detects the target, it also broadcasts a DETECTION message. When CS1 receives the second message from MS2 and if threshold $\mathrm{M}$ is set to 2 , then it actuates its camera to localize the target. In this case, the target is in its FoV. CS2 retrieves the real target's coordinates and sends them only to MS1 via LOCALIZATION message. In the following step, CS2 activates its camera after the reception of the DETECTION messages from MS2 and MS3 but the target is not in its FoV. In this case, MS2 is responsible for assigning approximate target coordinates using previous equations.

Successively, MS1 and MS2 send the results to the sink (we suppose that all nodes have a route toward the sink).

The added value of the protocol EAOT consists in giving the target coordinates closest to its real position and in the worst case the approximate coordinates can be generated. In addition, the visual tracking is exploited with minimal energy consumption.

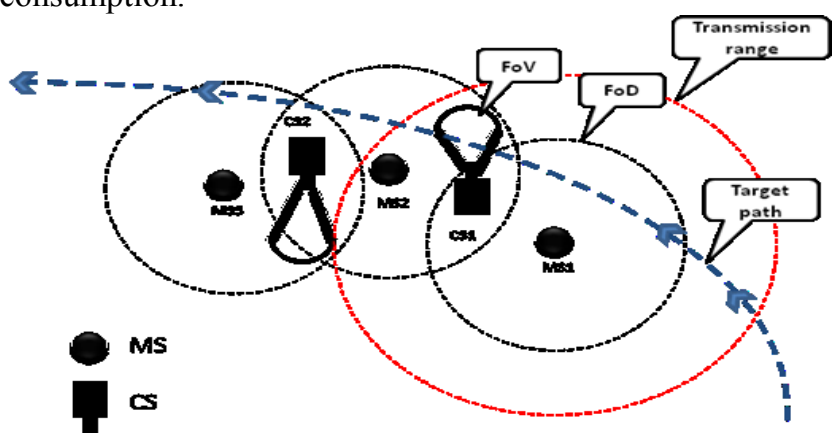

Figure 2. An example of object tracking with EAOT

EAOT is illustrated in figure 3. We present two flowcharts: one for MS nodes and the other one for CS nodes. As explained before, the object tracking process starts when an MS detects the target. Each MS that has the target in its FoD broadcasts a DETECTION message. If it receives a LOCALIZATION message, it extracts the target coordinates and transmits them directly to the sink. Otherwise, after a predefined period of time $\mathrm{T}$ corresponding to the total time of transmitting/processing a DETECTION message, localization process, transmitting/processing a LOCALIZATION message and the travel time of the target between two nodes. The MS acts like a fusion center and handles the localization. It calculates the approximate coordinates. The obtained results are then transmitted to the sink by the MSs. The sink handles the reconstitution of the trajectory by piecing together the received coordinates.

Figure 3 (B) illustrates the CS flowchart. When the CS receives at least $M$ DETECTION messages: $M$ plays an important role in tracking accuracy, when $M$ increases the tracking accuracy also increases. This CS activates its camera to capture images of the target and checks if the target is in its FoV using background subtraction [12]. If so, the concerned CS performs the localization via any existing solution [13] and sends it to the first MS. We suppose that this calculated coordinates are exact and error-free. Finally, it disables the camera. 


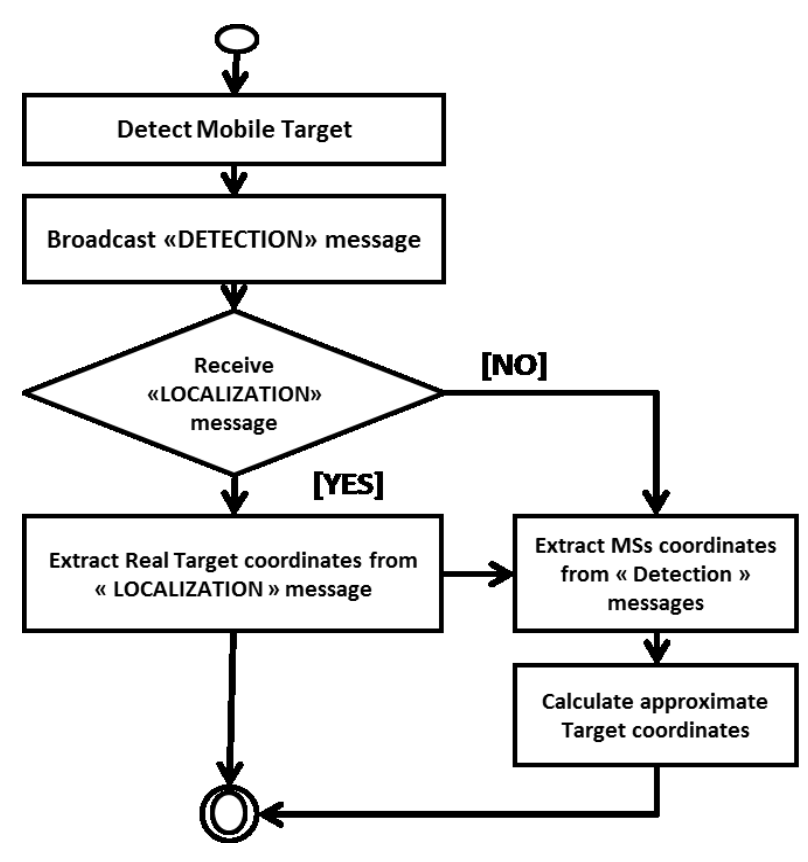

A. MS flowchart

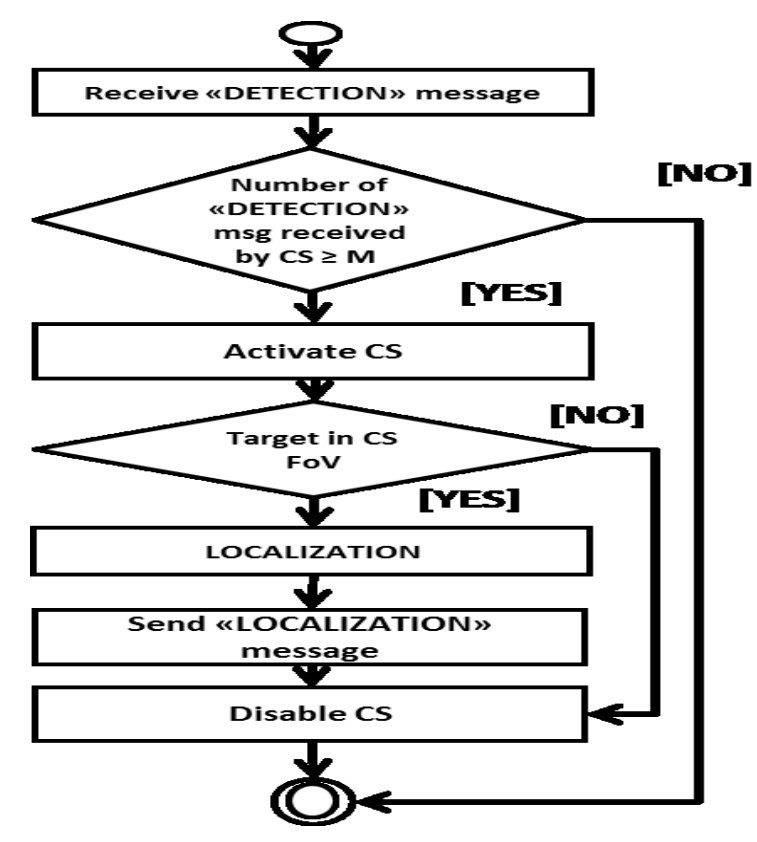

B. CS flowchart

Figure 3. EAOT flowchart

\section{PERFORMANCE EVALUATION}

In this section, we present the simulation settings, evaluation metrics and we discuss the obtained results.

\section{A. Simulation setting}

We have used the NS-2 simulator [14] in order to evaluate EAOT. In all scenarios, the nodes have been randomly placed in a rectangular two dimensional area. The parameter values of the simulation are summarized in Table 1.
TABLE 1. SIMULATION PARAMETER

\begin{tabular}{|l|l|}
\hline Mac Layer protocol & IEEE 802.15 .4 \\
\hline Access Mode & CSMA/CA non-beaconed \\
\hline Number of nodes & $20,30,40,50$ \\
\hline Area size & $100 \times 100 \mathrm{~m}^{2}$ \\
\hline Target speed & $1,38 \mathrm{~m} / \mathrm{s}$ (pedestrian) \\
\hline Simulation Time & $150 \mathrm{~s}$ \\
\hline Transmission range & $3 \mathrm{~m}$ \\
\hline Detection range (D) of MS & $15 \mathrm{~m}$ \\
\hline Depth of view (V) of CS & $20 \mathrm{~m}$ \\
\hline $\begin{array}{c}\text { Angle of view of CS } \\
\text { Size of message }\end{array}$ & $\pi / 3$ \\
\hline $\begin{array}{c}\text { M (number of activation } \\
\text { messages) }\end{array}$ & $2 \mathrm{messages}$ \\
\hline $\begin{array}{c}\text { Life span of “Detection " } \\
\text { message } \boldsymbol{T}\end{array}$ & $20 \mathrm{~s}$ \\
\hline
\end{tabular}

EAOT with different distributions of nodes is compared to two other solutions (Table 2). In both of these only CSs are used. The first one is the naive solution which consists in deploying CSs. These CSs are always in active mode: capturing/processing multimedia streaming. The second one is the OCNS (Optimal Camera Node Selection), the operating principle of OCNS [7] is explained in section II.

\begin{tabular}{|l|l|}
\multicolumn{2}{|c}{ TABLE 2. SCENARIOS } \\
\hline EAOT1 & $30 \% \mathrm{MS} / 70 \% \mathrm{CS}$ \\
\hline EAOT2 & $50 \% \mathrm{MS} / 50 \% \mathrm{CS}$ \\
\hline EAOT3 & $70 \% \mathrm{MS} / 30 \% \mathrm{CS}$ \\
\hline naïve & $100 \% \mathrm{CS}$, always in active mode \\
\hline OCNS & $100 \% \mathrm{CS}$, solution proposed in \\
& {$[7]$} \\
\hline
\end{tabular}

\section{B. Simulation results}

Three metrics are used to compare our mechanism to other solutions: tracking precision, number of messages exchanged and energy consumption. Another metric is applied only for EAOT distributions: spatial average deviation.

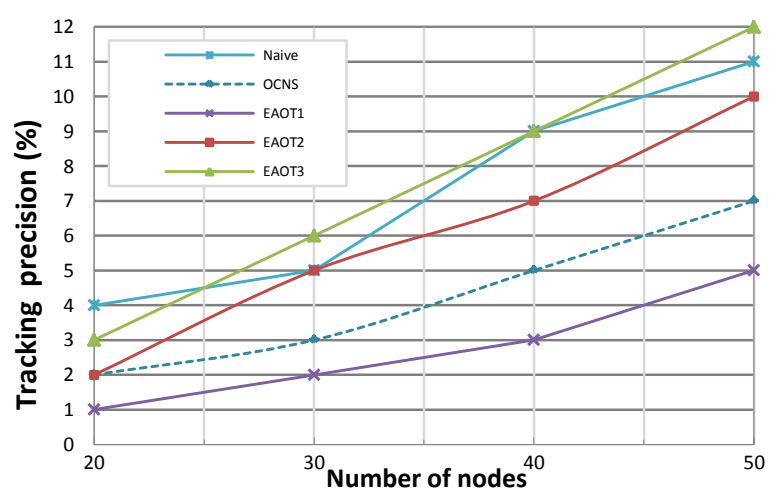

Figure 4. Tracking precision/number of nodes

TABLE 3. REAL TARGETCOORDINATES \%

\begin{tabular}{|l|c|}
\hline & Real target coordinates \% \\
\hline EAOT1 & $74 \%$ \\
\hline EAOT2 & $66,66 \%$ \\
\hline EAOT3 & $41 \%$ \\
\hline
\end{tabular}




\section{1) Tracking precision}

We measure it by considering the number of points collected for a defined trajectory. One point is represented by a two dimensional coordinate. For Naive and OCNS the retrieved points are the only points where we have an image of the target; we call them real target coordinates. In our solution the retrieved points can be a real target coordinates (extracted from the captured image) or an approximate target coordinates obtained from MS (equation 1 and 2). Figure 4 shows the average number percentage of retrieved points for every scenario depending on the number of nodes.

$100 \%$ represents the ideal tracking precision: for a pedestrian, the best number of retrieved points is 1 every meter.

Table 3 shows the real target coordinates percentage depending on the MS/CS distribution. We note that the best tracking accuracy is obtained by the naive approach because all CS nodes are always running and the visual target detection has a positive impact on the precision. Among the three EAOT schemes, EAOT2 represents the best compromise between real target coordinates and approximate ones and has a better target precision than OCNS. EAOT3 also has more retrieved points than OCNS but more approximate target coordinates. The main reason is the distribution of MS/CS. In EAOT3, we have more MS so it results in more approximate coordinates. EAOT1 has fewer points because there are not enough MSs to activate the CS.

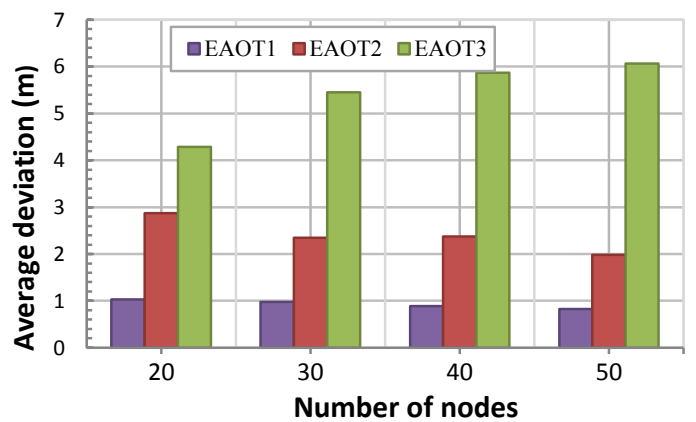

Figure 5. Average deviation

\section{2) Spatial average deviation}

This metric represents the average deviation of the obtained trajectory vs. the real one. It is obtained by calculating the average of the distances between approximate target coordinates and real ones.

We suppose that the coordinates calculated by the CSs are exact and error-free. So, they are not considered in average deviation results. Distance $d$ is calculated as follows:

$$
\mathrm{d}=\sqrt{\left(\mathrm{X}_{\mathrm{c}}-\mathrm{X}_{\mathrm{r}}\right)^{2}+\left(\mathrm{Y}_{\mathrm{c}}-\mathrm{Y}_{\mathrm{r}}\right)^{2}}
$$

(Xc, Yc) are the calculated coordinates and (Xr, Yr) are the real ones. Average deviation cannot be considered as tracking precision metric because it is only applied to EAOT schemes. It is the only solution where target coordinates can be real or approximate. Figure 5 shows the average deviation depending on the number of nodes and on the distribution. The results are obtained by calculating the distance between every retrieved point of EAOT schemes and the corresponding one in a real trajectory.

We observe that EAOT1 has the best results compared to other EAOT distributions due to the greater number of CSs than in other schemes. The average deviation of EAOT3 increases with the number of nodes. The main reason is the increase of MS: the more MSs we have, the more approximate coordinates are obtained.

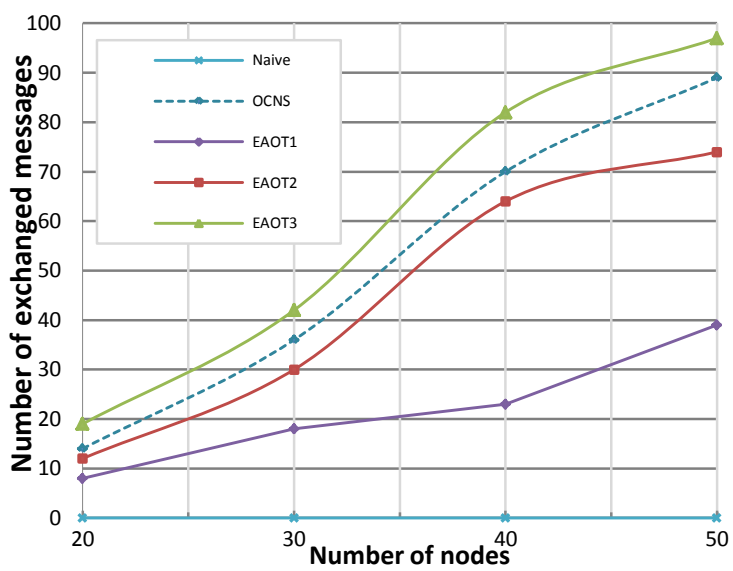

Figure 6. Number of exchanged messages according to the number of nodes

\section{3) Number of exchanged messages}

We suppose that the message size is the same in all solutions. This metric shows the number of cooperative messages exchanged. For EAOT distributions it consists of both DETECTION/LOCALIZATION messages exchanged during the simulation. In OCNS, in addition to the cooperative messages, we add the messages of the wake up algorithm which does not exist in EAOT. Indeed, we assume that the MSs are always in active mode. Moreover, the messages transmitted to the sink are neglected in this metric for all solutions.

Figure 6 shows the average number of messages depending on the number of nodes. We remark that the number of exchanged messages increases with the number of nodes whatever the scenario. Only EAOT3 has more exchanged messages than OCNS, because of the high number of MSs which broadcast "DETECTION" messages when they detect the target.

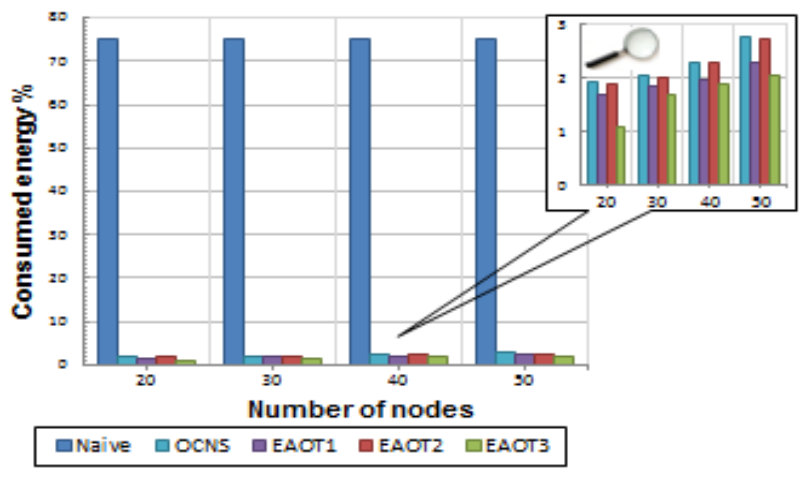

Figure 7. Average energy consumption according to the number of nodes 


\section{4) Energy consumption}

The percentage of energy consumption includes the energy consumed by cooperative message exchanges and the activation of MSs and CSs during one simulation. Figure 7 shows the average energy consumption percentage of the entire network depending on the number of nodes. $100 \%$ represents the total network energy available before the simulation starts. It is obvious that Naive consumes much more energy than the other solutions. Due to the use of scalar sensors for the detection, whatever the distribution of CS/MS EAOT always consumes less energy than OCNS. These results confirm the efficiency of EAOT for energy aware object tracking.

From the presented experiments and results, we can deduce that:

- Naive is an unrealizable solution. Despite the fact that it performs the tracking with the best precision, this solution is too costly and considerably reduces the network's lifetime.

- EAOT2 is the distribution which presents the best trade-off between tracking accuracy and energy consumption. It performs tracking with better precision, less energy and a smaller number of exchanged messages than OCNS.

- The simulation results can be improved by optimizing the node's positioning.

\section{CONCLUSION AND FUTURE WORKS}

Saving energy is a crucial issue in WMSNs whatever the research topic and the application field. Multimedia tracking of mobile targets being a costly application in terms of energy, the need for a low cost solution increases. In this paper, we proposed a new approach using Heterogeneous Wireless Sensor Networks (HWSNs). The main objective of our solution called EAOT for Energy Aware Object Tracking is to handle the tradeoff between tracking precision and energy consumption.

We conducted some simulations with different scenarios and distributions of MS/CS to evaluate our solution. The results show that EAOT increases the tracking precision and reduces the energy consumption compared to OCNS where only CSs are used. The simulations performed also show that the distribution of $\mathrm{MS} / \mathrm{CS}$ has an un-negligible impact on the obtained results. Our future works will consist in improving EAOT by adding the targets' identification and classification as well as the multi-target tracking.

\section{REFERENCES}

[1] I.F.Akyldiz, T.Melodia,K.R Chowdhury," A survey on Wireless Multimedia Sensor Networks", in Computer Networks 51 (2007) 921960

[2] G.Jin, X,Lu, M,Park, "Dynamic clustering for object tracking in wireless sensor networks », in Proc.3rd Int .Symp. UCS, Korea, 2006.

[3] W. Heinzelman, A. Chandrakasan, and H. Balakrishnan; "EnergyEfficient Communication Protocol for Wireless Microsensor Networks". Proceedings of the Hawaii Conferenceon System Sciences, Jan. 2000
[4] P. Pahalawatta, T. N. Pappas, and A. K. Katsaggelos, "Optimal sensor selection for video-based target tracking in a wireless sensor network," in Proc.IEEE Int. Conf. Image Process, Singapore,2004

[5] Z.R.Zaidi, B.LMark, "Mobility Tracking based on Autoregressive Models"l, in IEEE Transactions on mobile Computing, Vol 10,January 2011.

[6] F.Zaho, J.Liu, J.Liu, L. Guibas, and J. Reich "Collaborative Signal and Information Processing:An Information Directed Approach" Proceedings of the IEEE 91 (8) (2003)

[7] L.Liu, X,Zhang," Optimal Node Selection for Target Localisation in Wireless Camera Sensor Netwoks", in IEEE Trans. Vehicular Technology Vol. 59, No. 7, September 2010.

[8] P.Kulkarni,D.Ganesan,P.Shenoy,Q.Lu,"SensEye: A Multitier Camera Sensor Network", in ACM International Conference on Multimedia (2005)

[9] A.Newell,K,Akkay,'Distributed Collaborative camera actuation for redundant data elimination in Wireless Multimedia Sensors", networks,Ad Hoc Netwoks, June 2011

[10] R.Stolkin, I Florescu, "Probability of detection and optimal sensor placement for threshold based detection systems".(2007)

[11] T.He,C.Huang,B.M.Blum,J.A.Abdelzaher,"Range-free localization schemes for large scale sensor networks",in ACM Mobicom(2003)

[12] M.Piccardi," Background substraction", in IEEE Int.Conf.,Syst.,Man, Cybern. Oct. 2004

[13] H.Oztrarak,K.Akkay,A.Yazici,"Lightweight object localization with single camera in Wireless Multimedia Sensor Networks", IEEE GLOBAL COMMUNICATIONS CONFERENCE (IEEE GLOBECOM 2009)Honolulu, Hawai, USA, November 2009

[14] Ns-2, http://www.isi.edu/nsnam/ns 